\section{Cahiers de Narratologie}

Analyse et théorie narratives

$35 \mid 2019$

Le style comme événement

\title{
Fiction et identification : de la narratologie à la sémiotique contemporaine
}

\section{Christophe Gauld}

\section{(2) OpenEdition}

\section{Journals}

Electronic version

URL: http://journals.openedition.org/narratologie/9496

DOI: 10.4000/narratologie.9496

ISSN: 1765-307X

Publisher

LIRCES

\section{Electronic reference}

Christophe Gauld, «Fiction et identification : de la narratologie à la sémiotique contemporaine », Cahiers de Narratologie [Online], 35 | 2019, Online since 03 September 2019, connection on 15 November 2019. URL : http://journals.openedition.org/narratologie/9496

This text was automatically generated on 15 November 2019.

Article L.111-1 du Code de la propriété intellectuelle. 


\title{
Fiction et identification : de la narratologie à la sémiotique contemporaine
}

\author{
Christophe Gauld
}

L'auteur remercie pour son aide à la construction et à la relecture du texte le Dr Charles-Edouard Notredame, Hôpital Fontan, CHU Lille

\section{Introduction}

1 L'identification peut être conçue comme le fait d'emprunter, pour un temps, la perspective d'autrui, afin de mieux assoir son individualité propre. Dans ce mouvement, on relève ainsi une confusion identitaire partielle. Il y a confusion, car l'intimité des ressentis émotionnels semble touchée par la sensation d'autrui ; elle est partielle, car elle n'opère pas une «dissolution» de la représentation ou de la conscience du soi (Duval, 2009). Pour mieux comprendre l'autre, en se projetant émotionnellement, le corps et l'esprit "vivent» la sensation identifiée ${ }^{1}$. Cette identification, résultat empathique d'une projection émotionnelle, est avant tout un processus social. Elle fonde notre perception sociale et y assoit notre rôle. Si ce phénomène est quotidiennement vécu entre deux personnes réelles, il est permis de se demander ce qu'il en est de notre perception des personnages fictifs. En effet, nous savons bien qu'ils ne peuvent être saisis de la même manière que les personnes réelles dans le monde de la réalité quotidienne. Et en même temps, nous y sommes constamment confrontés, à travers différents médias, de la littérature au cinéma. Peutil seulement y avoir un lien identificatoire du sujet au personnage ? Et si cette relation existe, quelles en sont les conséquences sur les comportements de la personne qui s'identifie?

2 Cette notion est cruciale, parce que l'essence même des canaux par lesquels se déploie la fiction est d'émouvoir : le livre ou le film sont créés pour faire naitre des sensations chez leur public. Au moment de notre immersion dans l'univers qu'ils proposent, nous 
sommes saisis de ce qu'il y a de plus puissant dans l'acte humain de "contagion émotionnelle ", véritable ébranlement de nos craintes, de nos excitations, de nos joies, ou de notre désarroi. Pour mieux comprendre le fondement de cette notion dans le cadre de la fiction, nous allons prendre l'exemple de la cinématographie, canal privilégié pour la relative homogénéité de ses représentations. Après une présentation brève du concept, nous avons ainsi considéré le processus d'identification en le replaçant autour de trois axes : la recherche scientifique expérimentale, la sémiotique, et les interprétations hypothétiques de la catharsis telle qu'elle fut présentée initialement par Aristote. Nous allons donc étudier les processus de la narration en cela qu'elle s'exprime sous le prisme de l'identification. Nous n'allons donc pas aborder le vaste champ de la narratologie questionnant le rôle de l'invention contrefactuelle, la création de l'image cohérente du monde narratif, l'expérience esthétique, ni étudier la nature des émotions produites par un récit fictif, thèmes par exemple abordés dans le vaste champ de la narratologie cognitive.

\section{Identification et émotion}

3 La notion d'identification peut s'inscrire dans différents cadres. Elle peut être conçue comme un phénomène de contagion émotionnelle, correspondant au transfert d'émotions d'une personne émettrice à une personne réceptrice ; on retrouve ici l'idée d'une perte de distance entre soi et autrui (Decety, 2003). L'identification peut également être comprise comme capacité cognitive, permettant de se mettre « dans la peau » de l'autre. Cette seconde approche fait de l'identification la conséquence d'un processus cognitif nommé théorie de l'esprit (c'est-à-dire la capacité à adopter le point de vue d'une autre personne), dans sa composante dite "chaude», c'est-à-dire appliquée aux processus émotionnels. Dans ce second cas, le sentiment de l'autre n'a pas nécessairement à être vécu, car une « simulation incarnée » de son émotion suffit à déclencher la réponse empathique (Decety, 2006 ; Lamm et Decety, 2007). De près ou de loin, l'identification est un des processus permettant l'expression de l'empathie (conçue comme reconnaissance et compréhension des sentiments et des émotions d'autrui, et expression réactionnelle) ${ }^{2}$. Dans la suite de ce travail, nous allons plutôt nous attacher au mécanisme de production de l'identification qu'à la réaction émotionnelle qui en découle.

4 Suckfüll (Suckfüll, 2009) explique comment fonctionne le modèle de résonance identificatoire entre spectateur et fait fictif. Il décrit deux modes de réception des films : la réception émotionnelle, basée sur la confrontation des enjeux du spectateur et de la vie du personnage à l'écran (Vorderer, 1992); la réception analytique, définie comme une réflexion sur les conditions de production (réalisateurs, caméra, lieu de tournage, etc.), et provoquant une attirance pour le média impliqué et l'histoire qu'il dévoile. Suckfüll décrit également une réception basée sur la fonction d'évasion, qui donne le sentiment d'être présent dans le monde fictif décrit. Ces deux modes de réception permettent de comprendre qu'un film provoque l'identification en fonction des caractéristiques propres au personnage, mais également du fait des conditions techniques de réalisation. 


\section{Compréhension pratique des effets identificatoires liés à la réalisation cinématographique}

5 Quels sont les mécanismes sous-jacents à la réalisation, qui nous mettent dans cette état «d'acceptation» du film et de son univers? Selon Van Sijill (Van Sijill, 2006), spécialiste en analyse de scène, ces éléments sont classés en différents domaines :

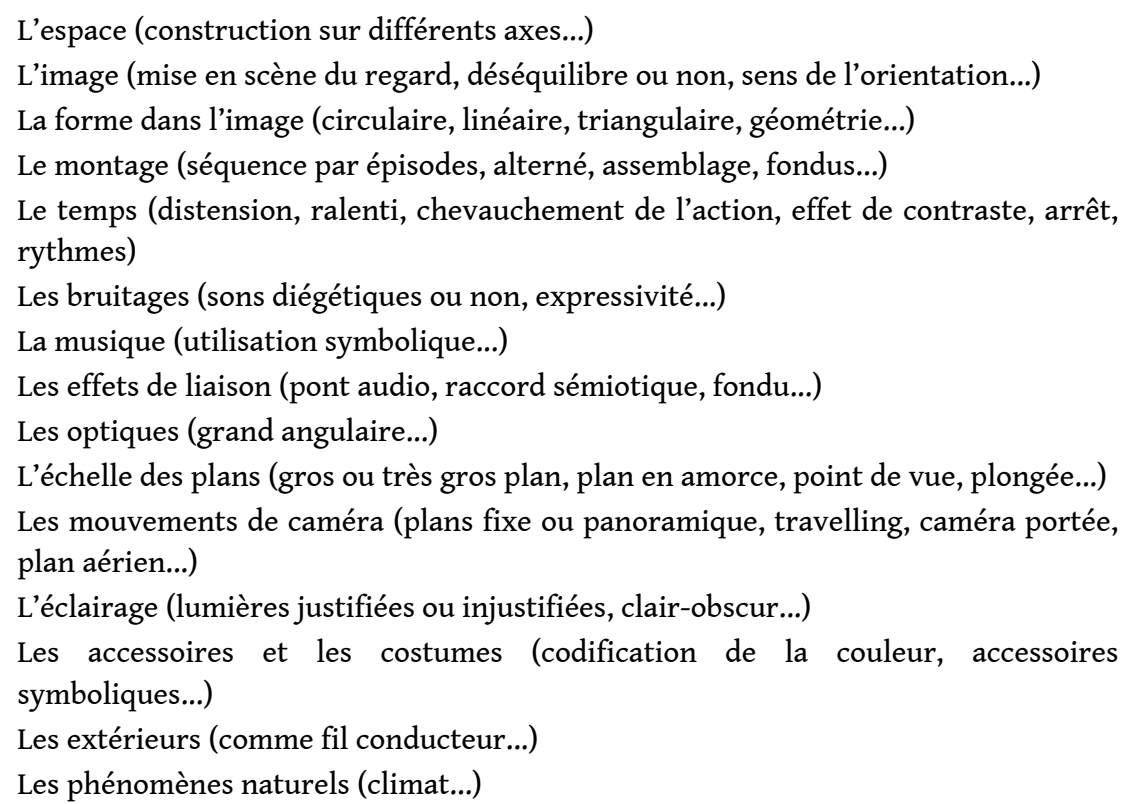

6 Il serait fastidieux de tous les détailler, mais de manière tout à fait empirique, à travers ces données, nous pouvons distinguer trois dimensions susceptibles d'induire une identification : l'image, le son, et la temporalité (ou rythme). Illustrons quelques-uns des éléments de cette liste, avant d'en offrir un commentaire. Certaines valeurs de plan ajoutent au dramatique de la scène narrative. La proximité du personnage augmente l'implication du spectateur, et un plan plus large entraine une «perte » de l'attention du spectateur dans l'environnement. De même, une contre-plongée entraine une "soumission" du spectateur au personnage, présentant donc une vision démunie, vulnérable, voire décomposée de celui-ci; une plongée donne au contraire une impression de "supériorité ». On pourrait encore citer la signification portée par les couleurs : des filtres blancs, jaunes, ou verts donnent une impression plus joyeuse de la scène, tandis que des filtres rouges, violets, ou bleus laissent un sentiment plus sombre (Heller, 2009). Dans L'oeil du photographe, on retrouve cette citation: «Le plan audessus de l'épaule peut permettre de manipuler le niveau d'identification du spectateur au personnage. Il suffit pour cela de bien contrôler l'angle de prise de vue. Plus l'angle épouse le point de vue du personnage situé au premier plan, plus la connexion émotionnelle et l'identification du public à ce personnage est intense. " (Freeman, 2017). Selon Flitterman-Lewis (Flitterman-Lewis, 1987), c'est la caméra qui, en donnant le point de vue à l'audience, détermine la cible de l'identification. Enfin, on pourra donner comme exemple l'exploitation du gros plan sur un visage, qui impose un maintien du spectateur dans l'humeur désirée ; le réalisateur pourra également utiliser le zoom pour augmenter l'expression jouée, et donc la communication de l'émotion au spectateur. La volonté de provoquer l'identification est ainsi un élément fondamental de la réalisation. 


\section{Quelle science de l'identification au cinéma?}

7 Focalisé sur les règles de composition d'un cadre, en ayant analysé un très large patrimoine cinématographique, Mercado a déterminé quels éléments sont habituellement mis en œuvre pour obtenir un impact significatif sur l'état émotionnel des spectateurs (Mercado, 2011). Certaines règles de composition sont devenues des standards: elles s'appliquent systématiquement à un type de plan singulier, pour induire une émotion particulière. La recherche cinématographique utilise d'ailleurs le système FACS (Facial Action Coding System) (Ekman et Friesen, 1984), qui permet d'associer une émotion (jouée par un acteur) au potentiel ressenti du spectateur. Le metteur en scène utilise cette "boite à outils ", habituellement appliquée au domaine de la recherche en sciences cognitives, avec pour but de provoquer de l'émotion dans son public. Heimann a également démontré que les indicateurs de l'activité sensorimotrice pouvaient varier en fonction du type de mouvement de la caméra (par exemple, zoom, steadicam, dolly), de la présence d'une bande-son, de bruitages, ou de lumières d'intensité et de teintes différentes (Heimann, 2016). De plus, l'activation des aires cérébrales liées à une observation est plus prononcée lorsque l'action perçue appartient au répertoire moteur de l'agent, et peut donc faire l'objet d'une réplication motrice, comme c'est le cas lorsque la scène est réaliste ${ }^{3}$. Grodal, nomme «l'attention particulière " (correspondant à un mécanisme d'attention focale), la technique qui permet d'amorcer l'attention du spectateur grâce à des signaux qu'il a déjà repérés auparavant. C'est le système de l'objet symbolique (dans une scène clé, les réalisateurs placent souvent, en effet, un objet qui a été porteur d'une valeur symbolique tout au long du fil de l'histoire) (Grodal, 2009). Enfin, tout un champ neuroscientifique (la narratologie cognitive, dont les relations avec la narratologie sont par exemple décrites dans Ryan, 2015) s'attache ainsi à définir comment nous pouvons ressentir des émotions pour les personnages fictifs, même si nous savons qu'ils n'apparaitront pas dans notre monde (Pichon et Vuilleumier, 2011). Peu de données sont cependant disponibles dans ce domaine, et elles manquent souvent de valeur méthodologique. On pourra par exemple citer le ciblage des neurones «canoniques", qui nous permettent la manipulation mentale des objets fictifs ou théoriques: cette fonction cognitive permettrait de «manier » mentalement le personnage fictionnel, tout en sachant qu'il ne peut apparaitre dans la réalité. (Speer, 2009 ; Rizzolatti, 1998).

\section{Sémiotique et cinéma}

8 Nous avons vu combien le travail de réalisation pouvait tenir une place importante dans le processus d'identification du spectateur au personnage. Cependant, l'origine contingente de ce mouvement empathique n'a pas été explicité. S'il est possible, et même conseillé, de s'attacher à ses semblables, est-ce seulement envisageable vis-à-vis d'un personnage de fiction? Il semble toutefois qu'une relation de confiance existe bel et bien entre le spectateur et le personnage, et ce lien semble être émotionnellement chargé. Simplement, qui ne s'est jamais senti proche d'un personnage de film? (Stein, 2009). Nous avons dit en introduction que l'identification se formait par nécessité de partage social. Qu'en est-il de notre identification au personnage fictif, qui ne semble pas transporter de valeur sociale? 
Or, l'empathie, condition nécessaire mais non suffisante à l'identification, est souvent exacerbée lors de la visualisation d'un film. Nous comprenons en effet intimement les ressentis du personnage, car le propre du film étant de pouvoir nous faire accéder à leur esprit. Un "bon film» est celui pour lequel nous vivons avec le héros "par procuration" (le "bon film " étant celui qui nous implique, nous immerge foncièrement). Ne tenons-nous pas même ces personnages pour " plus reels » que des personnes réelles, lorsque nous nous émouvons avec eux? Comme le dit Eco: «Beaucoup de lecteurs raffinés ne pleureront probablement pas sur le destin de Scarlett O'Hara mais ils auront été certainement choqués par le destin d'Anna Karénine » (Eco, 2010). Toutefois, il semble que si quelqu'un nous demandait si cette Anne Karénine était bien morte, la réponse serait systématiquement affirmative, et tout au plus avons-nous pu nous en émouvoir lors de la première lecture du livre de Tolstoï ; mais si jamais cette même personne nous demandait si notre petit(e) ami(e) était décédé(e), nous aurions sans doute un sursaut et éprouverions un bouleversement soudain. La question de l'émotion ressentie pour un personnage fictif semble ainsi complexe : qu'est ce qui fait que le sort de ce personnage-ci nous émeut, alors que, par exemple, des millions d'êtres bien réels meurent de famine dans le monde? (Johansen, 2002) $)^{4}$

Nous aimerions nous arrêter un instant sur les données qu'offre la sémiotique, c'est-àdire l'étude de la réception des signes et des symboles, et plus particulièrement de la sémiotique visuelle (promue par l'Association Internationale de Sémiotique Visuelle). Afin de mieux saisir ce lien entre réel et fictif, cette discipline propose l'introduction du concept d'OPE, pour Objets Physiquement Existants : Anna Karénine n'est pas un OPE, car elle dépend d'une conception mentale pour exister. Elle n'est pas « physique ». Il ne s'agira pas de considérer ici ce qu'est (ontologiquement) un personnage de fiction, mais en quel sens pouvons-nous parler de lui (et donc nous émouvoir) comme s'il existait (la sémiotique dirait « à quel genre de contenu » correspond l'expression Anne Karénine). Selon Searle, nous "signons un pacte tacite" avec l'auteur ou le réalisateur pour admettre que nous devons prendre au sérieux le personage qu'il met en scène; et en effet, nous ne le considérons jamais comme un "mensonge " (Searle, 1975). On dit que qu'Anna Karénine est fictionnellement vraie. Ainsi, lorsque nous acceptons ce monde fictionnel, nous pourrions être pris dans un monde narratif si absorbant et captivant que nous en oublirions nous trouver dans un monde fictif (ou " monde possible » selon Kripke (Kripke, 2007)). De plus, dans ce monde fictif dans lequel nous sommes absorbés, nous n'y sommes pas "recensés». Nous n'intervenons qu'en observateur. Notre engagement nous pousse alors à assumer temporairement la personnalité de quelqu'un d'autre. Suivre le fil d'une histoire revient à la reconstruire son univers ${ }^{5}$. D'un point de vue sémiotique, assumer l'autre correspond à l'identification au personnage de fiction. Selon Eco (Eco, 2010), nous persistons à tenir pour vrai qu'Anna Karénine s'est suicidée lorsque « l'hallucination visuelle » de l'histoire a cessé. L'identification ne cesse pas à la fin du film. Nous restons fidèles à ces compagnons narratifs. C'est ainsi que des personnages de fiction « réussis » peuvent constituer des exemples fondamentaux et des modèles pour notre condition réelle.

11 Cette approche théorique nous permet de comprendre la force d'imprégnation d'un univers fictif sur nos états psychologiques; il conviendra désormais d'observer, au plus près du psychisme, quels événements intérieurs mènent un sujet à éprouver des 
émotions devant cette fiction. En effet, s'il n'y trouve aucune nécessité sociale, qu'estce que le spectateur gagne à se déposséder de ses émotions à travers le média du film?

\section{Catharsis et cinéma}

12 Au deuxième paragraphe du sixième chapitre de La Poétique d'Aristote (Aristote, La Poésie, 1449 b 24-28), l'un des passages les plus commentés de l'auteur fait mention de la «catharsis » ${ }^{6}$. Dans son œuvre, Aristote propose régulièrement de considérer «la pitié et la frayeur » comme des éléments douloureux, et qui sont ici l'objet de cette épuration. Il semblerait alors que celle-ci permettrait la transformation de sentiments désagréables en plaisir, offrant une faculté paradoxale à cette mise en scène des passions (Dupont-Roc et Lallot, 1980).

Si la compréhension classique de la catharsis en fait un processus proprement moral, permettant de "guérir » de nos douleurs par la visualisation et le ressenti d'autres souffrances, nous nous rangerons plutôt aux côtés de Corneille (Corneille, 1660), qui se demande ce que pourrait bien "purger » une représentation d'Oedipe Roi. Notre désir d'inceste et de parricide disparaitrait donc après la représentation? Freud parlera de ce « bénéfice de plaisir qui nous est offert pour permettre la libération d'une jouissance supérieure émanant de sources psychiques profondes ", en en faisant une " décharge " désexualisée, permise par l'identification au personnage tragique, « projection idéalisée du moi ». Le mécanisme de cette faculté paradoxale serait, selon divers commentateurs, le fait de l'épuration des passions : celles-ci seraient isolées de leur contexte humain par le tragique de l'oeuvre, permettant une meilleure compréhension de leur essence, et nous libérant alors de leur poids (Aristote, La Politique). Mais tentons de comprendre le lien qu'entretient cette notion entre narration fictionnelle et émotion du spectateur.

Ricoeur reprendra cette notion de catharsis pour la replacer dans le rapport que l'œuvre entretient avec son récepteur. On retrouve ainsi, dans Temps et Récit (Ricœur, 1983) : «Parce que la tragédie met en scène une fiction, les événements douloureux, terrifiants, qu'elle donne à voir sur la scène produisent un tout autre effet que s'ils étaient réels. Chez le public, désengagé par rapport à eux, ils "purifient" les sentiments de crainte et de pitié qu'ils produisent dans la vie courante. S'ils les purifient, c'est qu'au lieu de les faire simplement éprouver, ils leur apportent par l'organisation dramatique une intelligibilité que le vécu ne comporte pas. Arrachées à l'opacité du particulier et de l'accidentel par la logique d'un scénario qui épure en simplifiant, condensant, systématisant, les souffrances humaines, d'ordinaires déplorées ou subies, deviennent dans le miroir de la fiction tragique objets d'une compréhension. ». Ricoeur se décale donc de la position sémiotique citée précédemment: il parle ainsi d'un « désengagement » du public par rapport à la fiction, en opposant cette dernière à la réalité 7 Selon lui, on ne vit pas de la même manière les événements fictionnels et réels. Cela ne signifie pas pour autant que l'oeuvre fictionnelle est moins puissante (ou moins " contagieuse ») que la réalité ; au contraire, elle pourrait comporter une dimension systématique, qui fournirait une composante morale et rationnelle plus aisée à maitriser. L'oeuvre permet l'élucidation de certaines pratiques.

Enfin, avec Hume, on pourra considérer la catharsis comme passage d'un seuil. Comme l'écrit l'auteur (Hume, 1742) : «De l'exemple du chatouillement, il ressort que, lorsque l'intensité du plaisir est poussée un peu trop loin, celui-ci devient peine, et que l'intensité de la peine, un peu tempérée, la transforme en plaisir. De là vient qu'il puisse 
exister une chose telle qu'une peine douce et agréable: c'est une peine affaiblie et diminuée. ». Hume admet que nous savons toujours que l'œuvre fictionnelle est irréelle ; la peine éprouvée étant alors réduite, elle est susceptible de se transformer en plaisir (passant « sous le seuil »). Le « tourment agréable » est la conséquence d'un mélange de larmes et de "délices", selon Hume. Mais dans cet assemblage, nous exprimons souvent la passion la plus importante, à savoir la peine (bien que cette valence émotionnelle puisse être différente en fonction de notre implication dans le film, et que la moitié du public pourrait sourire quand l'autre pleurerait).

Il est très intéressant de noter que Hume affirme que c'est bien « l'éloquence même avec laquelle la scène désolante est évoquée » qui fera naitre la joie, le "plaisir du coeur " à l'accueil d'une scène ou d'une situation désolante: cette "éloquence " correspond au jeu de la réalisation du film, en quelque sorte à la beauté avec laquelle est produit la scène du film. En somme, c'est l'absence d'indifférence qui nous affecte positivement (c'est-à-dire, selon Hume, "l'infusion d'un nouveau sentiment»). La passion douloureuse, lorsqu'elle est soulevée par la production cinématographique et artistique, est embellie et adoucie au point de nous fournir un réel plaisir, ou du moins un soulagement agréable.

Pour résumer, il semble que l'on peut distinguer trois étapes successives au processus d'identification :

l'identification proprement dite, qui se créé lorsque le spectateur admet (émotionnellement) se retrouver dans la même situation problématique que le personnage ;

la possibilité qu'il a d'éprouver des émotions par le biais du personnage (ce qu'il ne peut pas toujours faire dans la vie réelle), entrainant un effet cathartique ;

la prise de connaissance des solutions envisagées par le personnage (il développe ainsi des compétences pour trouver des solutions à des problèmes globalement similaires) (Giasson, 2013).

Pour mieux comprendre cet ensemble de processus dynamiques et dont la valeur n'est pas toujours évidente à saisir, nous allons détailler, dans un dernier paragraphe, comment les contenus identificatoires se positionnent en terme de valence émotionnelle, pour un sujet particulier.

\section{Valence émotionnelle et réaction empathique paradoxale}

Deux types méthodologiques d'études expérimentales témoignent de l'impact du film sur le spectateur: les media effect studies, qui considèrent les spectateurs comme des récepteurs passifs du média impliqué, et étudient le contenu du média lui-même ; et les reception audience studies, qui prennent en compte le rôle actif des spectateurs dans l'interprétation qu'ils donnent au contenu perçu.

Ces interprétations sont intimement associées à la connaissance préalable des caractéristiques du média par les individus, à leurs connaissances du monde social en général, et à leur style cognitif (tels que le style attributionnel décrit en sciences cognitives, qui consiste à attribuer schématiquement une valence positive ou négative à un événement particulier). Par exemple, la tendance à l'identification semble corrélée à un plus haut niveau d'empathie de la part du spectateur. Ainsi, comme le décrivait Hume, certains contenus cinématographiques vont permettre une émotion d'une 
certaine valence chez certains spectateurs, et d'une valence différente chez d'autres (en fonction du passage du « seuil »). De plus, le comportement empathique qui en découle diffère en fonction de chacun; autrement dit, l'identification entre spectateur et personnage, si elle se réalise, ne détermine nullement la nature des comportements sociaux. S'identifier à un modèle peut tout aussi bien conduire à l'imiter qu'à adopter un comportement contraire à celui observé. Dans ce dernier cas, ce comportement est nommé "paradoxal», parce que le spectateur préférera agir en contrepoint du personnage. L'ambivalence des processus identificatoires parait donc subtile. Ils se lient aux processus d'empathie, à l'attachement, à l'assimilation identitaire et à la contagion émotionnelle pour opérer également une différenciation, une coupure et une contrerésonance ${ }^{8}$. Till a prouvé que le spectateur était significativement plus «attire» pour un personnage qui vivait une situation réaliste, et qu'il s'y identifiait encore plus si celui-ci avait une histoire de vie globalement similaire à la sienne (Till, 2015). Press avait montré, de la même manière, que le réalisme d'un personnage ou d'une scène encourageaient l'identification (Press, 1989). Le réalisme, cependant, ne signifie pas nécessairement la similitude du comportement d'un personnage avec la vie réelle d'un spectateur (qui peut être une similitude avec un stéréotype, etc) (Huesmann et Taylor, 2006).

21 La contre-identification est un phénomène encore différent: il ne concerne pas la valence paradoxale du ressenti psychologique du spectateur (par exemple, éprouver de l'apaisement dans une démonstration de tristesse intense, propre au processus cathartique), mais bien plutôt la faculté cognitive et explicite de nier l'identification. De cette prise de position affirmée, en découle une volonté d'agir différemment (Newman, 1985).

\section{Conclusion}

Nous avons vu que l'attribution identificatoire dépendait particulièrement des caractéristiques personnelles du spectateur ${ }^{9}$ (ils pourraient correspondre à la variété des codes sociaux, aux valeurs culturelles, normatives, morales ou psychologiques).

Cette proximité identitaire entre spectateur et personnage est en tout cas recherchée dans la production cinématographique ${ }^{10}$, qui a développé un ensemble de critères permettant de produire de l'identification. La sémiotique, elle, nous propose de voir l'identification comme le fait d'assumer être entré dans l'univers du personnage de fiction. De son côté, l'effet cathartique décrit dans la philosophie herméneutique de Ricoeur et de Hume nous propose de considérer l'identification comme un processus de distanciation avec nos affects, permise par l'intensité narrative de oeuvre. Ces différents points de vue permettent une analyse fine de l'identification, processus narratologique par excellence du fait de sa dynamique supportée par la relation du spectateur et de oeuvre. 


\section{BIBLIOGRAPHY}

Aristote, Poétique, trad. J. Hardy, Gallimard, Paris, 1996.

Barbero (2005). Who's Afraid of Fictional Characters ?. Rivista di Estetica, Vol 66, 148-164.

Corneille (1660). Discours de la tragédie et des moyens de la traiter selon le vraisemblable ou le nécessaire, [dans :] Trois Discours sur le Poème dramatique ; éd. B. Louvat et M. Escola (1999). Flammarion, 95-100.

Decety (2003). Shared representations between self and others : A social cognitive neuroscience view. Trends in Cognitive Science, Vol 7, 527-533.

Decety et Jackson (2006). A social neuroscience perspective of empathy. Current Directions in Psychological Science, Vol 15, 54-58.

Dours (2003). Personne, personnage, les fictions de l'identité personnelle. Aesthetica, 208.

Dupont-Roc et Lallot (1980). Aristote, La Poétique. Éditions du Seuil, 53.

Duval et Piolino (2009). Le Soi à la loupe des neurosciences cognitives, De la conscience de soi à la conscience de l'autre. Psychol NeuroPsychiatr Vieil, Vol 7 (1), 7-19

Umberto Eco (2010). Quelques commentaires sur les personnages de fiction. Sociologies [En ligne], Dossiers, Émotions et sentiments, réalité et fiction.

Ekman et Friesen (1978). Basic and Applied Studies of Spontaneous Expression Using the Facial Action Coding System EM-FACS. Oxford university Press.

Farrugia (2008). Syndrome narratif et archétypes romanesques de la sentimentalité. Don Quichotte, Madame Bovary, un discours du pape et autres histoires, dans Farrugia et al., Émotions et sentiments. Une construction sociale, Paris, Éditions L'Harmattan.

Flitterman-Lewis (1987). Psychoanalysis, film and television. In R. C. Allen (Ed.), Channels of discourse: Television and contemporary criticism. Chapel Hill : University of North Carolina Press, $172-210$.

Freeman (2017). L'oeil du photographe, The Visual Story: Creating the Visual Structure of Film, TV, and Digital Media. Focal Press.

Giasson, (2013). La lecture de la théorie à la pratique. De Boeck, 277

Gibson (1966). The Senses Considered as Perceptual Systems, Boston : Houghton Mifflin. Journal of Comparative Neurology, Vol 251, 281-298.

Grodal (2009). Embodied Visions: Evolution, Emotion, Culture and Film. Oxford University Press, 324.

Hamon (1973). Un discours contraint. Poétique, Vol 16.

Heller (2006). Psychologie de la couleur, Pyramyd.

Huesmann et Taylor, (2006). The role of media violence in violent behavior. Annu Rev Public Health, Vol 27, 393-415.

Hume (1742). Essais esthétiques, trad. R. Bouveresse, Flammarion, 2000, 113-118.

Johansen, (2002). Literary discourse. A semiotic-pragmatic approach to literature. University of Toronto Press, 489. 
Keysers et Gazzola (2010). Somatosensation in social perception. Nature Reviews Neuroscience, Vol 11, 417-428.

Kripke et Lewis (1986). De la pluralité des mondes, Editions de l'Eclat.

Lamm et Decety (2007). The neural substrate of human empathy : effects of perspective-taking and cognitive appraisal. J. Cogn Neurosci, Vol 19, 42-58.

Le Manchec (2004). L'Adolescent et le récit. Pour une approche concrète de la littérature de jeunesse. L'ÉCOLE, 24.

Mercado (2011). L'art de filmer, Pearson, 2011.

Newman (1985). The Post-modern Aura. The Act of Fiction in an Age of Inflation, Evanston. Northwestern University Presses, 198.

Pichon et Vuilleumier (2011). Neuroimagerie et neuroscience des émotions. Medecine/sciences, Vol $27,8-9$.

Press (1989). Class and gender in the hegemonic process : Class differences in women's perceptions of television realism and identification with television characters. Media, Culture and Society, Vol 11, 229-251.

Radford (1975). How Can We Be Moved by the Fate of Anna Karenina ?. Proceedings of the Aristotelian Society, Vol 69, 67-93.

Ryan (2015). Narratologie et sciences cognitives : une relation problématique. Cahiers de Narratologie Analyse et théorie narratives [Internet], Vol 28.

Ricœur (1983). Temps et Récit. Vol I, 82.

Rizzolatti et al. (1998). The organization of the cortical motor system : New concepts. Electroencephalography and Clinical Neurophysiology, 106, 283-296.

Searle (1975). Speech acts and recent linguistics. Developmental Psycholinguistics and Communication Disorders, Vol 263.

Speer (2009). Reading Stories Activates Neural Representations of Visual and Motor Experiences. Psychol Sci., Vol 20, 989-999.

Stein et Vythilingum (2009). Love and Attachment : The Psychobiology of Social Bonding. CNS Spectr., 239-242.

Suckfüll (2009). Modes of reception for fictional films. The European Journal of Communication Research.

Sutrop (1999). Sympathy, Imagination and the Reader's Emotional Response to Fiction. Representations and Emotions. Gunther Narr Verlag, 29-42.

Sutrop (2000). Fiction and Imagination. Paderborn, Mentis.

Till (2015). Determining the effects of films with suicidal content : a laboratory experiment. The British Journal of Psychiatry.

Van Sijill (2006). Les techniques narratives du cinéma: Les 100 plus grands procédés que tout réalisateur doit connaître. Eyrolles.

Vorderer (1992). Audience involvement and program loyalty. Poetics, Vol 22, 89-98.

Allen, \& Smith (1997). Film Theory and Philosophy. Oxford University Press. 
Zwaan (1996). Processing narrative time shifts. Journal of Experimental Psychology : Learning,

Memory, and Cognition, Vol 22(5), 1196-1207.

\section{NOTES}

1. En d'autres termes, l'identification correspond à une «transcendance » du moi, qui accepte de faire corps avec l'autre, conçu en « résonance ».

2. Bien qu'ils ne fassent partie que d'une partie très relative de ces processus, nous soulignons l'importance des neurones miroirs dans ce processus émotionnel, retrouvés dans le développement de la théorie de la simulation incarnée (Keysers et Gazzola, 2010)

3. Rejoignant par là les travaux portant sur l'énaction développé par Damasio.

4. Le cinéma permet une identification qui semble, en fait, être une condition nécessaire de la vie en société : «Le travail du spectateur relève à la fois de l'interprétation (du personnage fictif) et de l'implication (dans le personnage qui prend vie dans et par le spectateur). Se faire autre, vivre par procuration, permet un apprentissage du soi et du monde » (Le Manchec, 2000).

5. Cette notion rappelle le «modèle de situation » décrit par Zwaan, désignant l'image du monde narratif construit au cours du déroulement de l'histoire, mis à jour lors des changements spatiotemporels (Zwaan, 1996).

6. «La tragédie est la représentation (mimèsis) d'une action noble, menée jusqu'à son terme, et ayant une certaine étendue, au moyen d'un langage relevé d'assaisonnements d'espèces variées, utilisés séparément selon les parties de l'œuvre; la représentation est mise en œuvre par les personnages du drame et n'a pas recours à la narration ; et, en représentant la pitié et la frayeur, elle réalise une katharsis de ce genre d'émotions ». Le terme katharsis est volontiers traité par " épuration », selon Dupont-Roc et Lallot.

7. Walton (Allen, 1997) prend l'exemple d'un spectateur au théâtre qui, pris de pitié pour l'héroïne menacée, saute sur scène pour la délivrer. Ce sauvetage échoue pourtant systématiquement, dans le monde de fiction comme dans celui de réalité ; ceux-ci n'ont en effet d'interaction qu'à travers la psychologie du spectateur (Dours, 2003)

8. Ce sont d'ailleurs ces effets « en dents de scie » qui font la consistance et l'intérêt de l'histoire narrative.

9. Par exemple, les personnages des manuels d'apprentissage scolaires sont régulièrement des enfants ressemblant aux élèves, pour que ceux-ci se reconnaissent dans ces « héros ».

10. Hamon a parlé « d'effet de vie » pour désigner l'ancrage du personnage dans une sociologie et une histoire, qui offrent une prévisibilité et une complicité au spectateur (Hamon, 1973).

\section{ABSTRACTS}

A travers une série d'observations et d'analyses concernant le statut du spectateur dans la fiction visuelle, la notion d'identification sera rediscutée sous le prisme de l'expérimentation cognitive, de la sémiologie, et de la philosophie herméneutique. L'identification, concept transdisciplinaire et polymorphe par excellence, est d'abord une relation qui assemble mondes réel et fictionnel. Elle trouve son origine et son point de chute dans la narratologie, de laquelle elle s'échappe 
cependant, afin d'opérer, par vicariance, une connexion particulière entre le spectateur et l'œuvre de fiction.

INDEX

Mots-clés: identification, cinema, narratologie, sémiotique, catharsis

Chronological index: XXIe siècle 